SCIENTIFIC LETTER

\title{
A 17 year experience of inappropriate shock therapy in patients with implantable cardioverter-defibrillators: are we getting any better?
}

\author{
C A Rinaldi, R D Simon, A Baszko, J Bostock, D Elliot, C A Bucknall, J S S Gill
}

Heart 2004;90:330-331. doi: 10.1136/hrt.2003.026195

Ins mplantable cardioverter-defibrillators (ICDs) are standard treatment for both secondary and primary prevention in patients with malignant ventricular arrhythmias. ${ }^{12}$ Inappropriate shock therapy (IST) occurs in a significant proportion of ICD patients, despite newer generation devices and algorithms specifically employed to try and eliminate this. ${ }^{3}$

The frequency of IST in patients implanted with cardioverter-defibrillators at this institution over a 17 year period from 1984 to 2001 was assessed. Predictive factors of IST were assessed, as was the susceptibility of certain subgroups to IST. Also, newer dual chamber devices were examined to see if they reduced IST.

\section{METHODS}

We retrospectively analysed data from 171 patients. Each patient had an ICD capable of storing electrograms that met the programmed criteria for detection (that is, fell within a ventricular tachycardia (VT) or ventricular fibrillation (VF) therapy zone). Full data regarding delivered therapy were available in 155 patients. We analysed stored electrogram data from the ICD. Clinical assessments were made by studying ECG data and inpatient records from the time of hospitalisation, to assess whether therapy was appropriate. Therapy was deemed inappropriate when delivered for rhythms that were not VT or VF. A probability value of $\mathrm{p}<0.05$ was considered significant.

\section{RESULTS}

IST occurred in 22 patients ( $14 \%$ ). Of these, 18 received IST for atrial arrhythmias (flutter/fibrillation) or sinus tachycardia. In the other four, IST was related to lead problems (lead fractures). The mean (SD) number of inappropriate shocks per patient was 4.1 (10) (range 1-49). Patients were subdivided into those with IST (group $1, \mathrm{n}=22$ ) and those without IST (group 2, $\mathrm{n}=133$ ) (fig 1 ).

The majority of patients presented with syncope or cardiac arrest. Both groups were matched for age (63 (12) $v 59$ (152) years, $\mathrm{p}=\mathrm{NS})$. Implant duration tended to be longer in group $1(1247(1282) \vee 779(851)$ days, $\mathrm{p}=0.12)$. Group 1 patients tended to have worse left ventricular ejection fraction (LVEF) (39 (15)\% $v 45$ (15)\%, p = 0.08). Most patients were in New York Heart Association (NYHA) functional class I/II, with a trend towards lower NYHA class in group 1 ( $36 \% v 18 \%, p=0.08)$. The incidence of surgical revascularisation and previous myocardial infarction (MI) were similar. Presentation arrhythmia was similar between the two groups (VT in $73 \%$ of group 1 patients $v 70 \%$ of group 2 patients, $\mathrm{p}=\mathrm{NS})$. Antiarrhythmic use was similar $(87 \%$ group $1 v 86 \%$ group $2, \mathrm{p}=\mathrm{NS}) ; 81 \%$ in group 1 were using a class III agent (amiodarone/sotalol) $v 75 \%$ in group 2 .

We identified three independent predictors of IST:

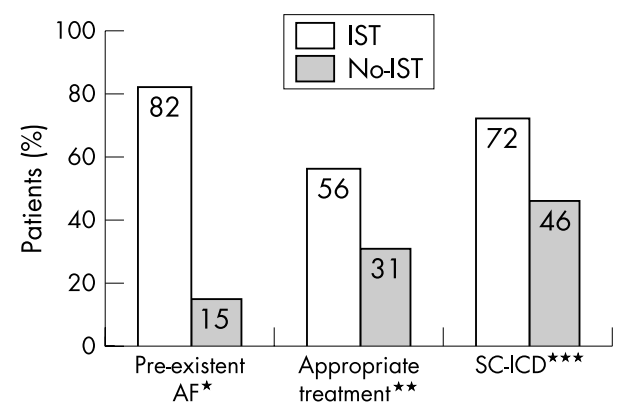

Figure 1 Incidence of the three predictors of inappropriate shock therapy (IST). Numbers quoted are percentages of pre-existing atrial fibrillation (AF), co-existing appropriate therapy, and single chamber implantable cardioverter-defibrillators (SC-ICD). ${ }^{*} p<0.001$, ${ }^{* *} \mathrm{p}<0.05,{ }^{* * *} \mathrm{p}<0.05$ all IST versus no IST.

- Pre-existing atrial tachyarrhythmias: $18 / 22$ (82\%) group 1 $v 20 / 133(15 \%)$ group 2 ( $p<0.0001$, relative risk (RR) 5.4, odds ratio (OR) 25.4)

- Coexistent appropriate therapy: 12/22 (56\%) group $1 v 4 \mathrm{l} /$ $133(31 \%)$ group $2(\mathrm{p}=0.049, \mathrm{RR} 1.76, \mathrm{OR} 2.69)$

- Single chamber (SC-ICD) device: there were 77 SC-ICDs $(50 \%)$ in total. Atrial fibrillation (AF) incidence was similar for both device types (27\% SC $v 22 \%$ dual chamber (DC), $p=N S$ ). Seventy two per cent of group 1 had an SC-ICD $v 46 \%$ of group 2 ( $\mathrm{p}=0.035$, RR 1.57, OR 3.09). IST was far more common with SC $v$ DC devices $(21 \% v$ $8 \%, p=0.02$, RR 1.6, OR 3.1) despite equal supraventricular tachycardia (SVT) discriminator programming in both groups. SC-ICDs tended to be older (mean implant duration $>1000$ days $v<600$ days for DC-ICDs $(\mathrm{p}<0.01)$.

Patients were divided into subgroups on the basis of coronary artery disease (CAD) and LV function. Those without $\mathrm{CAD}$ and $\mathrm{EF}<40 \%$-that is, dilated cardiomyopathy (DCM) - had an extremely high incidence of IST (50\%) compared to the other groups $(\mathrm{p}<0.05)$. AF incidence was

Abbreviations: $A F$, atrial fibrillation; $C A D$, coronary artery disease; DC-ICD, dual chamber implantable cardioverter-defibrillator: DCM, dilated cardiomyopathy; $\mathrm{EF}$, ejection fraction; ICD, implantable cardioverter-defibrillator; IST, inappropriate shock therapy; LV, left ventricular; MI, myocardial infarction; NYHA, New York Heart Association; SC-ICD, single chamber implantable cardioverterdefibrillator; SVT, supraventricular tachycardia; VF, ventricular fibrillation; VT, ventricular tachycardia 
also higher $(80 \%, \mathrm{p}<0.05)$ and a high proportion $(78 \%)$ had SC-ICDs.

\section{DISCUSSION}

We have identified a $14 \%$ incidence of IST, predominantly for atrial arrhythmias. We have identified several independent factors for IST: pre-implant AF, coexistent appropriate therapy, and implantation with an SC-ICD. Our results highlight a particular group which is at risk-that is, those with DCM. Our incidence of IST is relatively low compared to previous studies. Grimm reported an IST incidence of $22 \%$ in patients with ICDs implanted between 1983 and $1991^{3}$ and Nunain reported a $21 \%$ incidence of IST with newer third generation ICDs. ${ }^{4}$ During their lifetime more than $50 \%$ of ICD patients may develop AF, which is a well recognised risk factor for IST. ${ }^{3}$ The finding of increased coexistent appropriate therapy in IST subjects is not entirely clear, but may reflect poorer LV function, which itself predicts appropriate therapy. Similarly, AF itself may predispose to ventricular arrhythmias. The issue of DC-ICDs and SC-ICDs is important. Current evidence supports DC-ICDs in patients with a concomitant pacing indication, and a small number of studies have reported equal safety and effectiveness for SC and DC-ICDs. In our patient cohort DC-ICDs appeared superior to SC-ICDs $(21 \% \vee 8 \%, \mathrm{p}=0.02)$ and the $21 \%$ IST rate for SC-ICDs is surprisingly similar to earlier studies which included patients with older SC systems. ${ }^{3}$ Modern ICDs have specific algorithms to discriminate SVT from VT, but despite such algorithms there is still a relatively high proportion of IST caused by AF. In our patients, SVT discriminators were programmed on equally (85\% group $1 \mathrm{~V}$ $90 \%$ group $2, \mathrm{p}=\mathrm{NS}$ ) and in practice did not reduce IST except when used in conjunction with a DC-ICD. A recent editorial has suggested that DC-ICD discrimination algorithms are probably required for patients with frequent SVTs and that all ICD patients should receive a DC-ICD, which can detect and treat atrial arrhythmias with both antitachycardia pacing and cardioversion. ${ }^{5}$ Our finding of a particularly high incidence of IST in DCM patients is likely to be due to their high incidence of AF. Notably a large proportion of DCM patients had SC-ICDs and may have been denied the potential benefits of a DC-ICD.

A significant proportion of patients receive IST resulting in significant distress and potentially life threatening arrhythmias. Newer devices and discriminators decreased IST in our patients, but only when used in combination with a DC-ICD. Our data suggest that patients receiving an ICD should be assessed for atrial arrhythmias and that such patients may be better served with a DC-ICD.

\section{Authors' affiliations}

C A Rinaldi, R D Simon, A Baszko, J Bostock, D Elliot, C A Bucknall, J S S Gill, Cardiothoracic Centre, St Thomas Hospital, London, UK

Correspondence to: $\operatorname{Dr} C$ A Rinaldi, Cardiology Department, Royal Devon \& Exeter Hospital, Barrack Rd, Exeter, EX2 5DW, UK; c.a.rinaldi@btinternet.com

Accepted 8 September 2003

\section{REFERENCES}

1 The Antiarrhythmic versus Implantable Defibrillators (AVID) Investigators. A comparison of antiarrythmic drug therapy with implantable defibrillators in patients resuscitated from near-fatal ventricular arrhythmias. N Engl J Med patients resuscitated
1997; 337:1576-83.

2 Moss AJ, Hall WJ, Cannom DS, et al. Improved survival with an implanted defibrillator in patients with coronary artery disease at high risk for ventricular arrhythmia. N Engl J Med 1996;335:1933-40.

3 Grimm W, Flores BF, Marchlinski FE. Electrocardiographically documented unnecessary spontaneous shocks in patients with implantable cardioverter defibrillators. PACE 1992;15:1667-73.

4 Nunain SO, Roelke M, Trouton T, et al. Limitations and late complications of third generation implantable cardioverter defibrillators. Circulation 1995;91:2204-13

5 Santini M, Ricci R. Atrial fibrillation coexisting with ventricular tachycardia: a challenge for dual chamber defibrillators. Heart 2001;86:253-4. 